\title{
SISTEMAS DE INFORMACIÓN GEOGRÁFICA PARA OPTIMIZAR EL TRANSPORTE DE RESIDUOS A SITIOS DE DISPOSICIÓN FINAL EN EL ESTADO DE MÉXICO, MÉXICO
}

Geographic information systems for optimizing waste transportation to landfill sites in the State of Mexico, Mexico

\author{
Diana ENCISO GÓMEZ ${ }^{1}$, Pedro Hiram ANTONIO CERVANTES ${ }^{2}$, Fabián ROBLES MARTÍNEZ², \\ Enrique DURÁN-PÁRAMO ${ }^{2}$ y Diana Gabriela CASTRO-FRONTANA ${ }^{1 *}$
}

\footnotetext{
${ }^{1}$ Escuela Nacional de Ciencias Biológicas del Instituto Politécnico Nacional, Av. Wilfrido Massieu s/n, 07738 Ciudad de México, México

${ }^{2}$ Unidad Profesional Interdisciplinaria de Biotecnología del Instituto Politécnico Nacional, Av. Acueducto de Guadalupe s/n, 07340 Ciudad de México, México

*Autor de correspondencia: dgabyfrontana@yahoo.com
}

(Recibido enero 2017; aceptado mayo 2018)

Palabras clave: residuos sólidos urbanos, ciudad de México, estaciones de transferencia, sitios de disposición final, SIG

\section{RESUMEN}

El manejo de los residuos sólidos urbanos (RSU) generados en la Ciudad de México es un tema prioritario para el gobierno, ya que desde 2012 la ciudad no cuenta con un sitio de disposición final (SDF), motivo por el cual los RSU son llevados a diferentes sitios del Estado de México y Morelos. Esto implica mayores gastos y emisiones atmosféricas derivadas del transporte de los RSU a su sitio de disposición final. Una forma de mejorar es transportar los residuos de manera más eficiente a los SDF, puesto que la etapa de transporte representa hasta el $70 \%$ del costo total de manejo. Los sistemas de información geográfica (SIG) pueden usarse como herramienta para la optimización de rutas de transporte. En este trabajo se crearon bases de datos georreferenciadas con información de las estaciones de transferencia (ET) y de los SDF a los cuales llegan los RSU. Se mapearon los flujos de residuos desde las ET hacia los SDF y posteriormente, mediante una herramienta de análisis de redes usando SIG, se generaron rutas alternativas para el transporte de los RSU hacia cada SDF disponible. Se obtuvieron 66 rutas, de las cuales se eligió la más corta. Los resultados muestran que al menos ocho de 12 estaciones de transferencia no envían sus residuos al SDF más cercano, lo que implica un gasto mayor por concepto de transporte. El trabajo concluye que una manera de disminuir sustancialmente los costos es reorganizar las rutas para hacer más eficiente el transporte.

Key words: urban solid waste, Mexico City, waste transfer stations, landfill sites, GIS

\begin{abstract}
Solid waste management has become a major issue for the Mexico City's government because this city does not have its own landfill to dispose urban solid waste being generated on a daily basis. Currently, the solid residues are delivered to different sites within the State of Mexico and Morelos (neighbouring states). This decision
\end{abstract}


has resulted in very high transportation costs and emissions to the atmosphere. An improvement action would be to transport the solid waste in a more efficient way to the landfill sites, since this stage accounts for up to $70 \%$ of the total costs of the waste handling system. Geographical Information Systems (GIS) can be used as a powerful tool for the optimization of transportation routes. In this study, geodatabases containing information gathered at waste transfer stations (TS) and landfills (LF) were created and used to perform spatial analyses using GIS. Maps that show the destination of waste from TS in Mexico City to LF in the State of Mexico were created and then different transportation routes were generated from each TS towards each LF through a network analysis tool. More than 60 routes were obtained and compared with the distances to the current disposal sites. Optimal routes were then selected using the shortest-distance criterion. The results show that at least eight of the 12 transfer stations are not sending the waste to their nearest SDF. This study concludes that the optimization transfer stage is essential to reduce waste management costs and optimize transportation efficiency.

\section{INTRODUCCIÓN}

La generación de residuos sólidos urbanos (RSU) en la Ciudad de México, según el último inventario publicado por la Secretaría de Medio Ambiente de la Ciudad de México, asciende a 12893 toneladas diarias (SEDEMA 2015). Esto representa casi el 13\% del total nacional diario, ya que en la República Mexicana se generan aproximadamente 102895 toneladas diarias de RSU (INECC-SEMARNAT 2012). A partir de 1982, el Bordo Poniente, ubicado al oriente de la ciudad, fungió como sitio de disposición final de los RSU generados en la capital del país, convirtiéndose posteriormente en el principal SDF; si bien debía haber cerrado definitivamente el 31 de julio de 2008, el gobierno del entonces Distrito Federal no gestionó durante los años de su operación una solución real a la problemática tanto técnica como económica y social de los RSU a pesar de que el cierre era inminente (El Universal 2015).

Finalmente, el 19 de diciembre de 2011 se anunció de manera oficial el cierre del (sitio de disposición final (SDF) Bordo Poniente (La Jornada 2011a; El Universal 2011), y a partir de ese momento se agravó la problemática de la disposición final de RSU en la Ciudad de México. Durante los meses siguientes hubo poca información oficial de acceso público acerca del destino de las casi 13 mil toneladas diarias que se generaban en la ciudad (los inventarios oficiales de residuos de la Ciudad de México se publican casi un año después de la fecha a la que corresponden los datos reportados). Sin embargo, diversas notas periodísticas (La Razón 2011, La Jornada 2011b, Ciudadanos en Red 2012, Excélsior 2012, MTV 2013) dieron cuenta de la problemática que empezaba a crecer en la ciudad y en diferentes municipios aledaños, a partir de que el Gobierno del Distrito Federal (GDF) comenzó a enviar los RSU en camiones de gran capacidad a lugares más remotos donde habitualmente no circulaban, ocasionando malestar entre la población que habitaba cerca de los SDF. Estas notas periodísticas coincidían en que, a partir del cierre del Bordo Poniente, la transferencia de residuos se convirtió en el punto más crítico del tratamiento de residuos en la capital del país. La Ciudad de México (entonces Distrito Federal [DF]) contaba hasta 2012 con 13 estaciones de transferencia (ET) de RSU (SEDEMA 2013). Durante los días posteriores al cierre de Bordo Poniente las ET se vieron rebasadas por la cantidad de RSU que debían separarse en cada estación, con el fin de disminuir la cantidad de residuos que se enviaban a los nuevos SDF. Esto ocasionó problemas como retraso en la descarga de los camiones recolectores, incremento en los tiempos de traslado de hasta cinco o seis horas, aumento en las filas de camiones recolectores que esperaban entrar a las ET (con el consecuente descuido de su cobertura de recolección), malestar entre los vecinos de colonias aledañas, acumulación de residuos en diversas zonas de la ciudad, proliferación de olores y fauna nociva, etc. Sin embargo, en su Programa de Gestión Integral Residuos Sólidos 2016-2020, el GDF se había planteado como meta la generación cero de residuos (conocida como "Basura cero"), con el fin de aprovechar las cerca de 13 mil toneladas diarias de RSU que se producen, sin que hasta la fecha se haya podido demostrar (con base en los inventarios publicados por el propio gobierno de la ciudad), que se hayan reducido siquiera el $50 \%$ de los residuos generados (SEDEMA 2015, 2017).

Al respecto, la Secretaría de Medio Ambiente y Recursos Naturales (SEMARNAT) establece que la jerarquía deseable para el manejo de residuos es, en orden de preferencia: reducción en la fuente, la separación, la reutilización, el reciclaje, 
el coprocesamiento, el tratamiento, el acopio, el almacenamiento, el transporte y la disposición final como última opción (SEMARNAT 2012). Sin embargo, el GDF continúa enviando a disposición final el $62.75 \%$ de los residuos sólidos generados y además éstos son depositados en SDF ubicados en los estados de México y dos en el estado de Morelos (SEDEMA 2015).

Por otro lado, en su artículo segundo, fracción XVIII, la Ley General para la Prevención y Gestión Integral de Residuos (SEMARNAT 2003) define el concepto de manejo integral, el cual incluye las etapas de reducción en la fuente, separación, reutilización, reciclaje, coprocesamiento o tratamiento (biológico, químico, físico o térmico), acopio, almacenamiento, transporte, transferencia (opcional), disposición final y aprovechamiento energético cuando sea factible. Todo lo anterior, en un marco que se adapte a las condiciones y necesidades de cada lugar, cumpliendo con objetivos de valorización, eficiencia sanitaria, ambiental, tecnológica, económica y social. Sólo la etapa de recolección y transporte de los RSU puede representar hasta el $70 \%$ del costo total del sistema de manejo (SEMARNAT 2012, Betanzo et al. 2016).

Es claro que, a pesar de los esfuerzos emprendidos por el GDF, como la promulgación de la Ley de Residuos Sólidos local y su reglamento, la puesta en marcha de diversos programas de manejo (cartuchos de tóner, aceite vegetal, celulares, neumáticos, pilas) y otras acciones como el Reciclatrón, el Mercado del Trueque y el Mercado del Trueque escolar (SEDEMA 2015), éstos han sido insuficientes para transitar hacia un verdadero manejo integral de los residuos y para disminuir los problemas asociados a su mal manejo. Es importante destacar las implicaciones tanto técnicas como económicas y sociales de transportar grandes cantidades de residuos a sitios que no fueron diseñados para tal fin, ya que el envío a sitios cada día más lejanos es insostenible desde el punto de vista económico y ambiental. El presente trabajo analiza los flujos de residuos desde las ET hasta los SDF y propone rutas óptimas (basada en el criterio de distancia mínima) que puedan traducirse en reducciones en los costos de transporte de RSU.

Para ello se emplean Sistemas de Información Geográfica (SIG) como herramientas para la visualización de la información y el análisis espacial de datos, en este caso en las etapas de transporte y disposición final de residuos. Se utilizó información recabada en encuestas aplicadas directamente en las ET por integrantes del grupo de trabajo de este proyecto, correpsondiente al periodo en que no existió información pública acerca del destino de los RSU, e información de los inventarios de residuos sólidos de la Ciudad de México que fueron publicados a partir de finales de 2012, así como otras referencias que aportan evidencia sobre los lugares a los que fueron enviados los RSU en el periodo de diciembre de 2011 a 2014.

\section{MARCO DE REFERENCIA}

\section{El sistema "transferencia-disposición final" de RSU en la Ciudad de México}

El objetivo de las estaciones de transferencia de RSU es reducir el tiempo de traslado y descarga de los vehículos recolectores, para incrementar así la eficiencia del servicio de recolección. De acuerdo con el Inventario de Residuos Sólidos del Distrito Federal 2012, la ciudad contaba con 13 ET ubicadas en las delegaciones Álvaro Obregón, Azcapotzalco, Benito Juárez, Coyoacán, Cuauhtémoc, Gustavo A. Madero, Milpa Alta, Miguel Hidalgo, Tlalpan, Venustiano Carranza, Xochimilco e Iztapalapa (esta última es la única delegación que posee dos ET, debido a la alta generación de RSU en la Central de Abasto). Dichas estaciones se encargan de recibir los residuos de las 16 delegaciones políticas de la entidad para luego trasladarlos a camiones de mayor capacidad y llevarlos a seis SDF conocidos como La Cañada, Cuautitlán, El Milagro y Xonacatlán, en el Estado de México; y Cuautla y Tepoztlán, en el estado de Morelos (SEDEMA 2013).

Pero, incluso antes del cierre de Bordo Poniente, el entonces Distrito Federal ya enviaba una parte de sus residuos a otros SDF: La Cañada, 3 t/día; Cuautitlán, 102 t/día; El Milagro, 41 t/día; Xonacatlán, 33 t/día; Tepotzotlán, 32 t/día; Tultitlán, $2 \mathrm{t}$ /día, y Cuautla, $43 \mathrm{t}$ /día, sumando un total de $256 \mathrm{t}$ /día enviadas a disposición final fuera de la ciudad (Lámbarri et al. 2015). Esta cantidad se incrementó a $6153 \mathrm{t}$ /día en promedio (es decir, un incremento de $2400 \%$ ) después del cierre de Bordo Poniente (SEDEMA 2013). El cuadro I muestra las cantidades enviadas a disposición final fuera de la Ciudad de México antes y después del cierre de Bordo Poniente.

El cuadro II muestra la evolución de la infraestructura para el tratamiento, transporte y disposición final de RSU, reportada en los diversos inventarios de RSU publicados por la SEDEMA después del cierre de Bordo Poniente, así como información de las encuestas levantadas durante 2013 y 2014 en doce ET.

La ubicación de las ET y los SDF considerados para este trabajo se observan en las figuras $\mathbf{1}$ y $\mathbf{2}$, 
CUADRO I. CANTIDAD DE RSU ENVIADAA SDF FUERA DE LA CIUDAD DE MÉXICO, ANTES Y DESPUÉS DEL CIERRE DEL BORDO PONIENTE

\begin{tabular}{lcc}
\hline SDF & $\begin{array}{c}\text { Antes del cierre } \\
\text { del Bordo Poniente } \\
(2011)(\mathrm{t} / \text { día })\end{array}$ & $\begin{array}{c}\text { Después del cierre del } \\
\text { Bordo Poniente } \\
(2012)(\mathrm{t} / \text { día })\end{array}$ \\
\hline \multicolumn{3}{c}{ En el Estado de México } \\
\hline La Cañada & 3 & 2866 \\
El Milagro & 41 & 2191 \\
Cuautitlán & 102 & 613 \\
Xonacatlán & 33 & 287 \\
Tepotzotlán* & 32 & Sin información precisa* \\
Tultitlán* & 2 & Sin información precisa* \\
\hline \multicolumn{3}{c}{} \\
\hline Cuautla & En Morelos \\
Tepoztlán & No recibía RSU & 118 \\
Total & 256 & 68 \\
\hline
\end{tabular}

Fuente: elaboración propia a partir de datos de SEDEMA(2013) y Lámbarri (2015)

RSU: residuos sólidos urbanos, SDF: sitios de diposición final *Los sitios marcados con asterisco se reportan como sitios de prueba en el inventario 2012 de la SEDEMA, sin especificar más detalles de cuántos residuos recibían. En las encuestas realizadas durante 2013-2014 por el grupo de trabajo, la estación de transferencia Cuauhtémoc reportaba el envío de RSU al SDF de Tepotzotlán (lo cual corrobora que, durante algún tiempo, este SDF recibió residuos de la Ciudad de México)

respectivamente. Es importante destacar que para el análisis de rutas de este trabajo se utilizaron sólo los SDF ubicados en el Estado de México, que alguna vez recibieron RSU de la Ciudad de México.

\section{Aspectos técnicos de los vehículos que trasladan los residuos desde las ET hasta los SDF}

Como se mencionó anteriormente, el objetivo de instalar estaciones de transferencia en una localidad es hacer más eficiente el servicio de recolección de residuos y evitar que los camiones recolectores tengan que viajar grandes distancias hasta los SDF. Las estaciones se encargan de recibir los residuos de los camiones recolectores y posteriormente, una parte de ellos se canaliza a las plantas de separación. El material no recuperado se coloca en camiones tipo tráiler, conocidos como nodrizas, para finalmente ser transportados a diversos SDF. Las nodrizas pueden ser camiones de carrocería abierta o cerrada. Los primeros son los más utilizados; se trata de camiones tipo tráiler semirremolque con cajas de hasta $75 \mathrm{~m}^{3}$ y capacidad de transporte de 30 t. Los de carrocería cerrada son del tipo tráiler acoplado con una capacidad máxima de $50 \mathrm{~m}^{3}$ y transportan hasta $30 \mathrm{t}$ de residuos compactados, por lo que sólo pueden ser utilizados en ET equipadas con equipos compactadores (SEMARNAT 2012). Debido a las dimensiones de los camiones nodrizas, existen restricciones para su circulación, por lo que es deseable que circulen por calles bien pavimentadas y con un ancho adecuado para sus maniobras.

\section{Uso de los sistemas de información geográfica (SIG) para la creación y el análisis de rutas}

Los SIG son un conjunto de herramientas y procedimientos innovadores que usan datos espacialmente georreferenciados para resolver cuestiones

CUADRO II. INFRAESTRUCTURA PARA EL MANEJO DE RSU EN LA CIUDAD DE MÉXICO EN LAS ETAPAS DE TRANSFERENCIA, TRATAMIENTO Y DISPOSICIÓN FINAL

\begin{tabular}{|c|c|c|c|c|c|}
\hline Fuente & $\begin{array}{l}\text { Año de publicación/ } \\
\text { elaboración }\end{array}$ & $\begin{array}{l}\text { Estaciones de transferencia } \\
\text { (ET) en operación }\end{array}$ & $\begin{array}{l}\text { Plantas de } \\
\text { selección }\end{array}$ & $\begin{array}{l}\text { Plantas de } \\
\text { composta }\end{array}$ & $\begin{array}{l}\text { Sitios de Disposición } \\
\text { Final (SDF) }\end{array}$ \\
\hline \multicolumn{6}{|l|}{ Inventario de Residuos } \\
\hline $\begin{array}{l}\text { Sólidos del Distrito Fede- } \\
\text { ral } 2012\end{array}$ & 2013 & 13 & 3 & 10 & 6 \\
\hline \multicolumn{6}{|l|}{ Inventario de Residuos } \\
\hline \multicolumn{6}{|l|}{ Sólidos de la Ciudad de } \\
\hline México 2013 & 2014 & 13 & 2 & 7 & 5 \\
\hline \multicolumn{6}{|l|}{ Encuestas levantadas en } \\
\hline $12 \mathrm{ET}$ & 2013-2014 & 12 & - & - & 5 \\
\hline \multicolumn{6}{|l|}{ Inventario de Residuos } \\
\hline Sólidos CDMX 2014 & 2015 & 12 & 2 & 8 & 5 \\
\hline \multicolumn{6}{|l|}{ Inventario de Residuos } \\
\hline Sólidos CDMX 2015 & 2016 & 12 & 2 & 8 & 5 \\
\hline
\end{tabular}

Fuente: elaboración propia a partir de inventarios oficiales y encuestas RSU: residuos sólidos urbanos, SDF: sitios de disposición final, ET: estaciones de transferencia 


\section{Ubicación de las ET en la Ciudad de México}

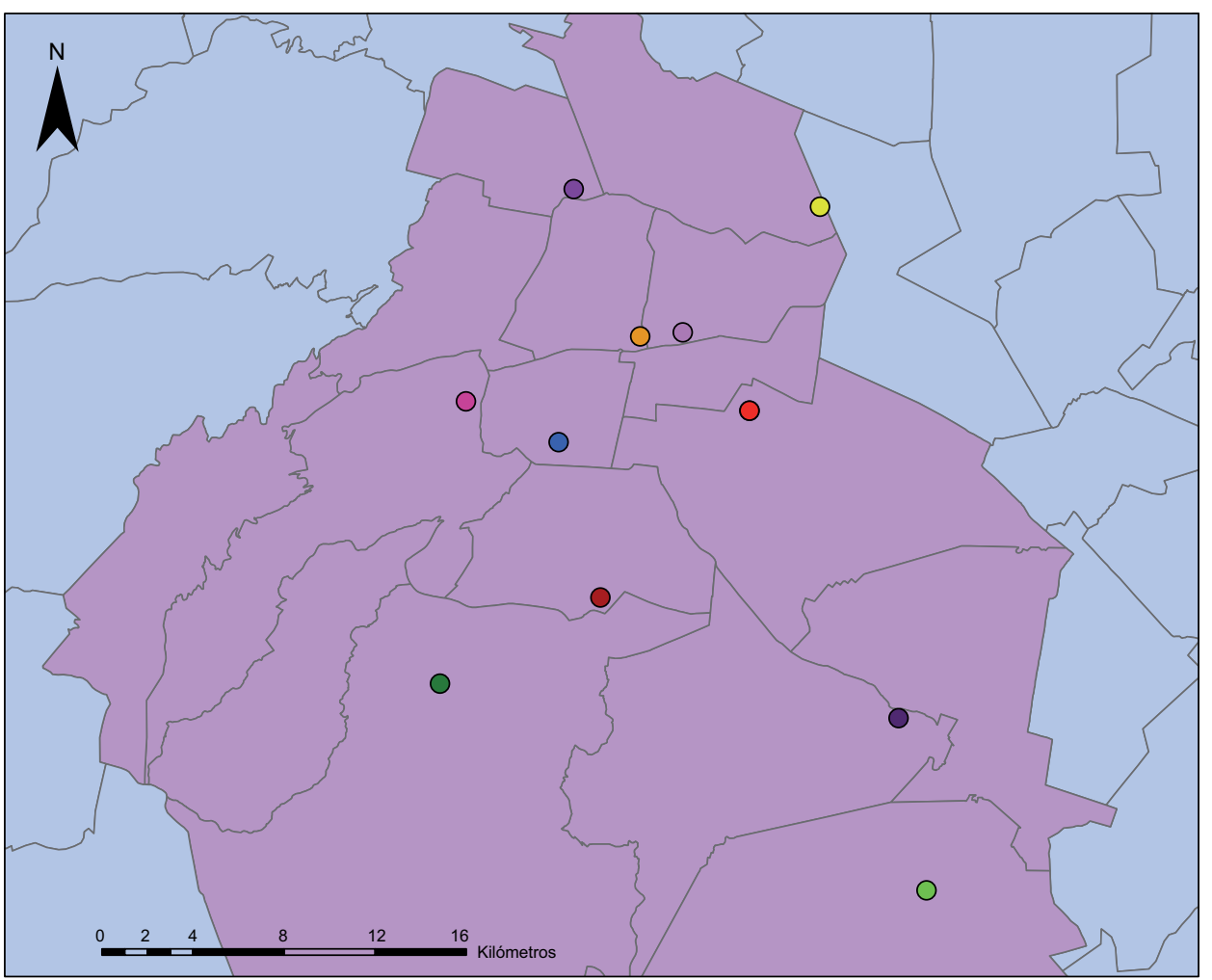

Alvaro Obregón

Azcapotzalco

Benito Juárez

Central de Abastos

Coyoacán

Cuauhtémoc

Gustavo A. Madero

- Iztapalapa

Milpa Alta

Tlalpan

Venustiano Carranza

Xochimilco

Ciudad de México

Edo. de México

Fig. 1. Ubicación de las estaciones de transferencia (ET) en la Ciudad de México (entonces Distrito Federal). Elaboración propia a partir del Inventario de Residuos Sólidos del Gobierno del Distrito Federal 2012

Ubicación de los SDF que recibían RSU de la Ciudad de México

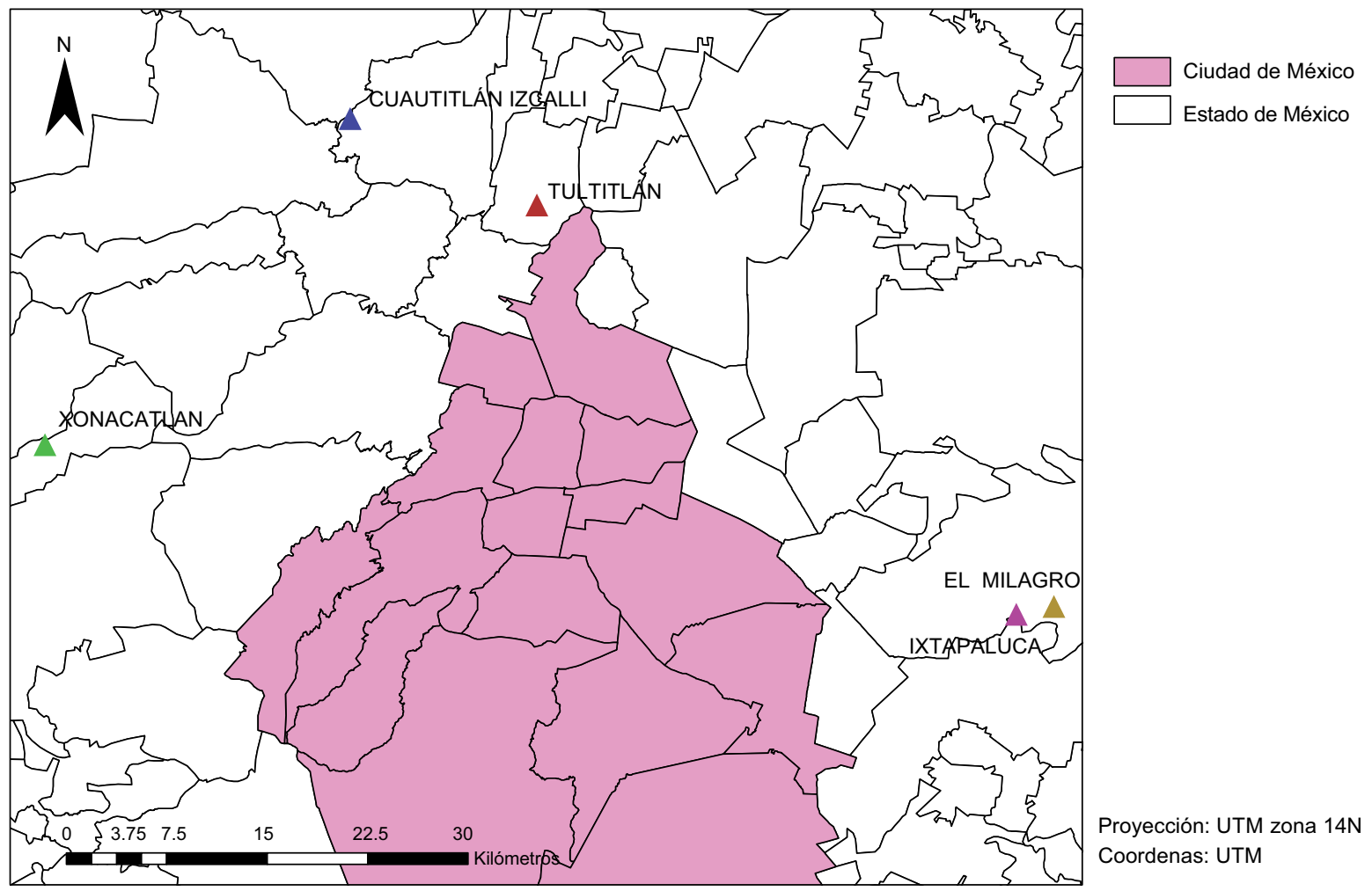

Fig. 2. Ubicación de los sitios de disposición final (SDF) en el Estado de México que recibieron residuos sólidos urbanos (RSU) de la Ciudad de México en el periodo 2011-2014. Fuente: elaboración propia a partir de encuestas e inventario de residuos publicados por la SEDEMA 
como la ubicación de instalaciones para proyectos ambientales (Kanchanabhan et al. 2010). El mundo real puede representarse en los SIG mediante dos modelos diferentes: vectorial y ráster. El primero representa los datos de la realidad por medio de puntos, líneas y polígonos, en tanto que el segundo se divide en filas y columnas, generando celdas en las cuales se encuentra y representa información del territorio donde cada celda puede almacenar una cualidad de la zona representada. La información en este formato se obtiene mediante imágenes digitales capturadas por satélites, o bien digitalizando un mapa o fotografía mediante el escaneo de imágenes. En los SIG el espacio geográfico georreferenciado se encuentra representado por medio de capas, las cuales se almacenan en un formato específico propio del software en el que se trabaja. Cada capa contiene uno o más atributos (características) del medio o de alguna temática en especial.

Una de las herramientas de los SIG es el análisis de redes (network analyst), el cual permite la generación de rutas a partir de dos puntos en una red de líneas en cuyas intersecciones se generan nodos. El fundamento del network analyst es la teoría de grafos, término matemático que se utiliza para designar un conjunto de puntos unidos entre sí por segmentos que pueden representar un proceso o relación funcional de cualquier tipo, pero que centra su atención en las relaciones topológicas entre sus elementos. Mediante la aplicación de esta teoría en los SIG es posible asociar redes de transporte o circulación con estructuras sencillas (abstractas) de nodos y arcos conectados; es decir, pueden asociarse fácilmente con objetos geográficos de la vida real, tales como generación de rutas de transporte, rutas críticas, entre otras (Cardozo 2009).

Existen diferentes criterios para la generación de rutas, entre otros los que destacan (Sendra et al. 2012):

- Criterio de distancia más corta de un punto a otro.

- Criterio de menor tiempo de viaje entre dos puntos.

- Criterio de menores gastos de peaje.

- Criterio de jerarquía de las calles.

El objetivo del presente estudio es crear una base de datos georrefenciada con los datos de los flujos de RSU de la Ciudad de México desde las ET hacia los diferentes SDF en el Estado de México, para visualizar estos flujos y realizar análisis espaciales con el fin de encontrar las rutas óptimas a los SDF usando el criterio de distancia mínima.

\section{MATERIALES Y MÉTODOS}

Para la creación de la base de datos georreferenciada se utilizó el software ArcView 3.2 ${ }^{\mathrm{a}}$, mientras que para realizar el análisis de redes usando SIG se utilizó el programa ArcGIS 10.2.2 (Hita 2009).

\section{Creación de la base de datos georreferenciada y creación de la red}

Para la creación de esta base de datos fue necesario definir el datum (D ITRF 1992) y proyectar los datos al sistema UTM zona 14N (correspondiente a la zona geográfica donde se encuentra el área de estudio) para el cálculo de distancias. Además, fue necesario determinar las capas necesarias para la elaboración de la red (capa de vías de comunicación) así como la capa de curvas de nivel (para visualizar la elevación de las ET y los SDF). En este caso la red debe entenderse como el conjunto de vértices (calles) y nodos (intersección de las calles, puntos de inicio [ET] y puntos de llegada [SDF] de los vértices), sobre la cual se realizará la medición de distancias y la creación de rutas por parte del programa ArcGIS.

La capa de vías de comunicación se obtuvo mediante descarga gratuita de una combinación de cartas de datos vectoriales disponibles en la página de Instituto Nacional de Estadística y Geografía (INEGI). Se realizó la unión de cartas vectoriales en escala 1:50 000 hasta cubrir la zona de estudio (Zona Metropolitana del Valle de México) y formar una sola capa de vías de comunicación (caminos).

Se hizo una depuración de la capa de caminos eliminando aquellos campos (columnas) con información irrelevante (claves, códigos, etc.) y se enriqueció la tabla de atributos con información necesaria para la elaboración posterior de la red para la generación de rutas: sentido de las calles, jerarquía y velocidad promedio. También fue necesario uniformar y clasificar la capa de caminos por tipo de calles de acuerdo con la clasificación del INEGI (1998), que especifica seis rubros para las vías de comunicación terrestre:

- Calle de primer orden: vialidad definida para el tránsito vehicular de una zona urbana, la cual puede estar representada por bulevares, periféricos, ejes viales, viaductos y avenidas principales.

- Calle de segundo orden: vialidad definida para el tránsito vehicular de una zona urbana, la cual puede estar representada por avenidas y calles de menor importancia que las definidas para calles de primer orden. También son vialidades principales dentro de localidades pequeñas que sirven de enlace entre carreteras. 
- Calle de tercer orden: vialidad definida para el tránsito local o peatonal dentro de una zona urbana. Ejemplos de estas son callejones, cerradas y privadas.

- Calle de cuarto orden: vialidad definida para el tránsito local dentro de áreas diversas.

- Carretera: vía de comunicación terrestre cuya estructura consta de un terraplén, obras de arte y revestimiento de asfalto o concreto, para el tránsito de vehículos con o sin pago de peaje.

- Terracería: vía de comunicación terrestre cuya estructura consta de obras de arte, revestimiento de arena y grava compactada para tránsito de vehículos.

Esta clasificación sirvió también para discriminar los caminos por los cuales no debería transitar un camión nodriza y hacer el cálculo de rutas exclusivamente sobre caminos de primer y segundo orden, así como carreteras, eliminando así los caminos por los que no es factible ni deseable que circulen los tractocamiones que transportan los RSU.

Es importante considerar que el software genera las rutas a partir de la red que se forma a través de los nodos que intersectan a las calles involucradas, por lo que la distancia final real de cada ruta puede variar respecto a la distancia calculada por el software. Este fenómeno ocurre en todas las calles con vuelta y se aprecia mejor en la figura 3.

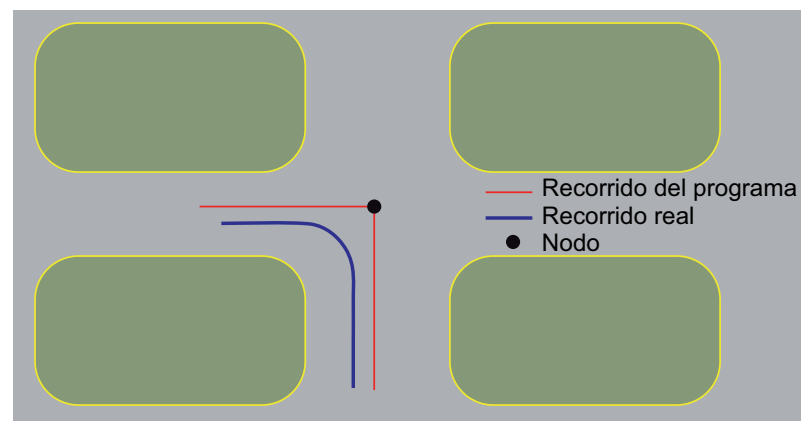

Fig. 3. Comparación del recorrido generado por el software SIG con el recorrido real. Fuente: elaboración propia

Otros nodos (puntos) involucrados en el análisis de redes son precisamente los correspondientes a los puntos de ubicación de las ET y los SDF. La capa vectorial con la ubicación de las ET y los SDF se construyó a partir de las coordenadas tomadas in situ durante las encuestas realizadas por el grupo de trabajo. Todos los insumos creados se exportaron a formato shapefile (.shp) y a una base de datos geográfica (geodatabase) para su posterior manipulación en el programa ArcGIS.

\section{Creación de las rutas desde cada ET hasta los SDF}

Una vez generada la red en el programa ArcGIS (ArcMap), se generan las rutas mediante la herramienta Network analyst (análisis de redes) con la opción "nueva ruta". Posteriormente, con la herramienta de ubicación de red se seleccionan los puntos de partida y llegada para la ruta y se elige la opción "resolver". Como resultado se genera una ruta con información como longitud calculada, asignación de colores, simbología, etc. El punto de inicio de cada ruta está dado por la ubicación de la ET y el SDF como punto final. Este paso se repitió considerando las 12 ET como puntos iniciales y cada uno de los SDF como puntos finales. Es importante mencionar que en esta etapa se descartó la ET de Miguel Hidalgo, ya que no fue posible levantar encuestas en el lugar, debido a que al momento de realizarlas ya se encontraba fuera de operación.

Finalmente, para determinar la ruta óptima entre las obtenidas, se consideró otro parámetro: altitud. Para ello se usaron las capas en formato vectorial de curvas de nivel de la Zona Metropolitana del Valle de México (ZMVM), clasificando la información por rangos de altitud mediante un color asignado a cada rango. De esta manera se puede distinguir visualmente la diferencia de altitud en diferentes sitios; se sobreponen las capas anteriores con la última para seleccionar aquellas rutas que, además de menores distancias, presenten menores cambios de altitud.

\section{RESULTADOS}

Primeramente, en el cuadro III se muestran los resultados de las encuestas realizadas durante el periodo 2013-2014, las cuales complementan los datos reportados en los inventarios de residuos sólidos de la SEDEMA. Esta información se complementa con la figura 4, en la que se representan los flujos de RSU de las ET hacia los diferentes SDF, tanto de la Ciudad de México como del Estado de México.

Como puede observarse, el primer lugar al que la mayoría de las ET enviaban sus RSU es el SDF localizado en Ixtapaluca, con 10 rutas; en segunda posición el SDF conocido como El Milagro, con seis rutas, seguido de los SDF de Cuautitlán Izcalli y Tepotzotlán, con una ruta cada uno. Dos de los SDF, Xonacatlán y Tultitlán, no cuentan con información que indique que alguna ET envía sus residuos a estos 
CUADRO III. RESULTADOS DE LAS ENCUESTAS LEVANTADAS EN LAS DISTINTAS ET DURANTE EL PERIODO 2013-2014

\begin{tabular}{lccc}
\hline ET & $\begin{array}{c}\text { Número de unidades } \\
\text { recolectoras que llegan } \\
\text { a la ET al día }\end{array}$ & $\begin{array}{c}\text { Número de tractocamiones } \\
\text { que trasladan RSU de las } \\
\text { ET a los SDF al día }\end{array}$ & $\begin{array}{c}\text { Destino de } \\
\text { los RSU }\end{array}$ \\
\hline Álvaro Obregón & 260 & 30 & Sin información \\
Azcapotzalco & 150 & 21 & La Cañada \\
Benito Juárez & 217 & 20 & La Cañada \\
Central de Abastos & 180 & 22 & La Cañada \\
Coyoacán & 325 & 30 & Ixtapaluca \\
Cuauhtémoc & 190 & 17 & La Cañada, el Milagro, Cuautitlán \\
& & & Izcalli y Tepotzotlán \\
Gustavo A. Madero & 350 & 16 & La Cañada y el Milagro \\
Iztapalapa & 172 & 31 & La Cañada \\
Milpa Alta & 45 & 5 & La Cañada y el Milagro \\
Tlalpan & 124 & 14 & La Cañada y el milagro \\
Venustiano Carranza & 215 & 12 & La Cañada y el Milagro \\
Xochimilco & 80 & 14 & La Cañada y el Milagro \\
\hline
\end{tabular}

Fuente: elaboración propia

RSU: residuos sólidos urbanos, SDF: sitios de diposición final, ET: estaciones de transferencia

Disposición de los RSU de la Ciudad de México a los SDF en el Estado de México
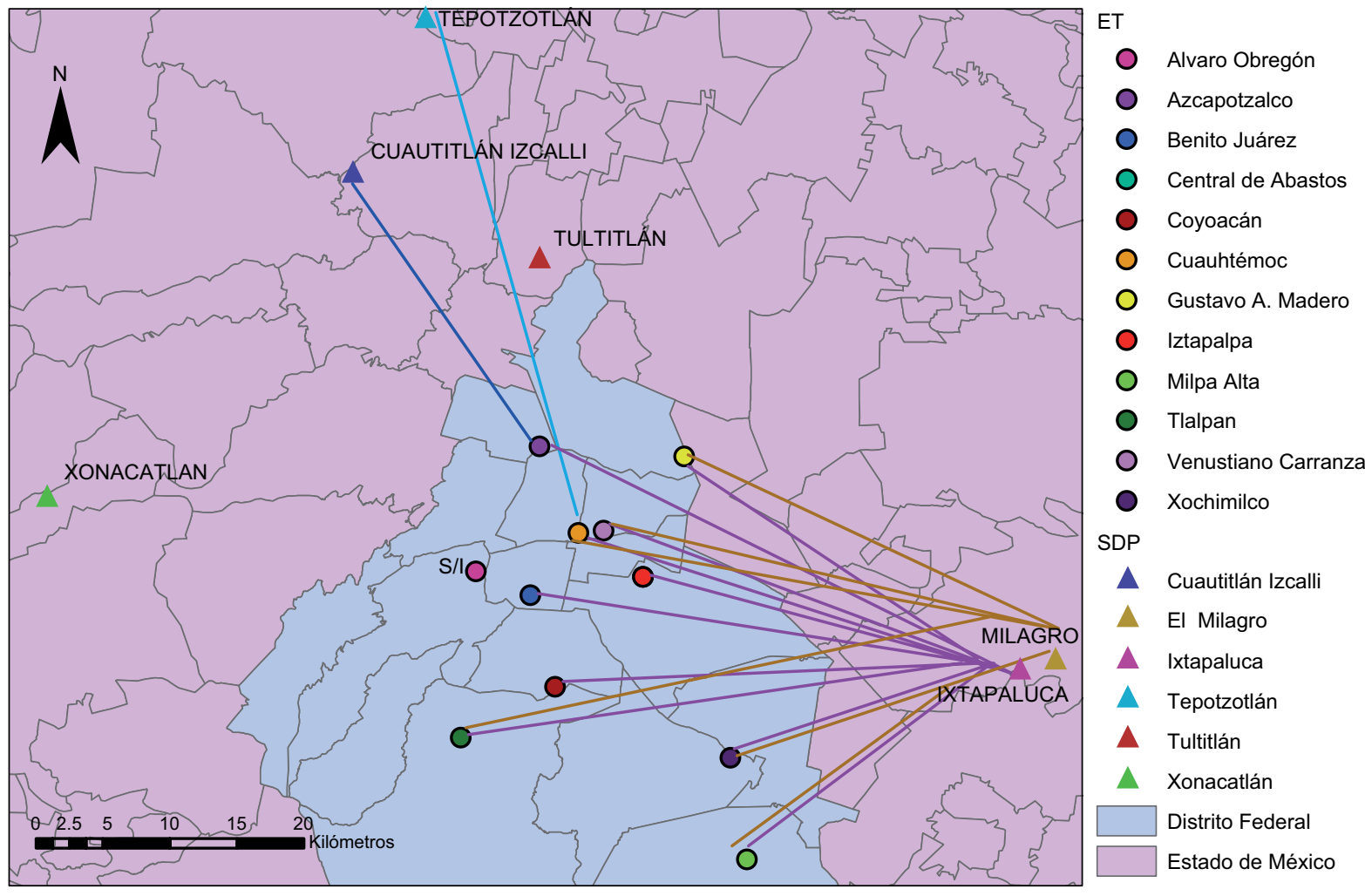

Fig. 4. Flujo de residuos sólidos urbanos (RSU) a estaciones de transferencia (ET) de la Ciudad de México y a sitios de diposición final (SDF) del Estado de México. Fuente: elaboración propia a partir de encuestas recabadas en 2013-2014 
dos sitios; sin embargo, mediante encuestas se sabe que sí recibieron residuos de la Ciudad de México durante el periodo de estudio (Lámbarri 2015) y por lo tanto se incluyeron en el análisis de rutas.

Con ArcGis se generó un SIG que incluye las 12 ET de la Ciudad de México y seis SDF del Estado de México; con la herramienta Análisis de rutas del mismo software, se crearon 72 rutas desde cada ET hasta cada SDF. Debido a que las ET de Central de Abastos e Iztapalapa tienen prácticamente la misma ubicación geográfica, el número de rutas se redujo a 66, ya que estas dos ET se consideraron como un mismo nodo; esto también se aplicó a los SDF de Ixtapaluca y La Cañada, debido a su cercanía geográfica. La determinación de la ruta óptima se basó en la distancia (planimétrica) más corta que existe desde cada ET hacia un SDF, aunque también se consideró el cambio de altitud en el terreno. Se crearon mapas de distancias desde cada ET hacia cada SDF, con seis rutas propuestas para cada estación. La figura 5a, b muestra un ejemplo de las rutas generadas para cada ET hacia los diferentes SDF sobre la red de caminos construida para tal fin.

El cuadro IV muestra los resultados de distancias planimétricas calculadas para las doce ET. En la columna SDF se muestra en negritas el SDF al que se enviaban los RSU desde cada ET, acorde con las encuestas y según el inventario 2012 de SEDEMA. De acuerdo con los datos recabados, una misma estación de transferencia puede enviar sus RSU a uno o más SDF. Por otro lado, en la columna Distancia calculada se muestra en negritas la distancia más corta. Cuando el SDF actual coincide en la ruta de la distancia óptima, se marca con un asterisco (*), lo que significa que la ET enviaba sus RSU al SDF más cercano durante el periodo de estudio.

De acuerdo con los resultados mostrados en el cuadro IV, sólo cuatro ET envían sus residuos al SDF más cercano (óptimo), mientras que ocho lo hacen a sitios no óptimos.

\section{DISCUSIÓN}

Con las capas y rutas obtenidas mediante el SIG, se realizó un análisis para determinar tanto la ruta óptima basada en el criterio de distancia mínima, como aquellas con menores variaciones en altitud, entre ET y SDF. Un ejemplo es el caso de la ET Gustavo A. Madero, cuyo SDF más cercano sería el de Tultitlán, a una distancia de $27.82 \mathrm{~km}$. Sin embargo, los datos recabados (Cuadro III y SEDEMA 2013) muestran que sus RSU se enviaban a tres SDF:
Cuautitlán, La Cañada y El Milagro, aunque no se posee información sobre las rutas específicas de los vehículos. Empleando el SIG creado en este estudio, se calcularon las distancias aproximadas a esos SDF, obteniéndose $37.34,34.55$ y $38.32 \mathrm{~km}$, respectivamente. Comparando las distancias, se observa que existe una diferencia de 6.7 a $10.5 \mathrm{~km}$ entre el SDF óptimo y los SDF utilizados para enviar los RSU durante el periodo de estudio.

Los resultados muestran también que sólo cuatro ET (Central de Abasto, Iztapalapa, Milpa Alta y Xochimilco) enviaban sus RSU al SDF más cercano u óptimo, aunque Milpa Alta también podía optar por el SDF ubicado en Cuautla, pero éste no se consideró en el estudio. Por lo anterior es notorio que sólo las ET Central de Abasto e Iztapalapa enviaban sus RSU al SDF más cercano.

El cuadro $\mathbf{V}$ muestra el resumen de los sitios más cercanos a cada ET, empleando el criterio de distancia mínima obtenida mediante la herramienta Análisis de redes. Es importante destacar que este análisis sólo consideró los SDF del Estado de México que recibieron RSU de la Ciudad de México durante el periodo de estudio. Para las ET ubicadas al sur de la ciudad es posible que el SDF de Cuautla sea el más adecuado.

Como puede observarse en el Cuadro V, para la mayoría de las estaciones de transferencia (Álvaro Obregón, Azcapotzalco, Benito Juárez, Coyoacán, Cuauhtémoc, Gustavo A. Madero, Tlalpan y Venustiano Carranza) el SDF óptimo resultó ser el de Tultitlán, lo cual puede atribuirse a que dichas ET están ubicadas en la parte centro y norte de la ciudad. Además, las vías de acceso para este sitio no tienen grandes cambios de altitud. El hecho de que un solo SDF haya resultado el de menor distancia para tantas ET puede no ser conveniente, ya que el SDF de Tultitlán se diseñó para recibir menos de la mitad de los RSU generados en la Ciudad de México. De hecho, Tultilán recibió muy pocos residuos de la capital del país y en los inventarios de SEDEMA se reportaba como un destino de prueba; sin embargo, los resultados indican que Tultitlán podría considerarse como un lugar adecuado para un nuevo SDF interestatal, dada su cercanía con ocho de las ET ya existentes. Esto requeriría estudios más detallados para seleccionar un terreno que cumpla con la NOM-083-SEMARNAT-2003, además de que sería necesario establecer un acuerdo entre el gobierno de la Ciudad de México y el del Estado México que permita un verdadero manejo integral de residuos a nivel metropolitano. Los RSU generados en la Ciudad de México aunados a los generados en el Estado 
(a)

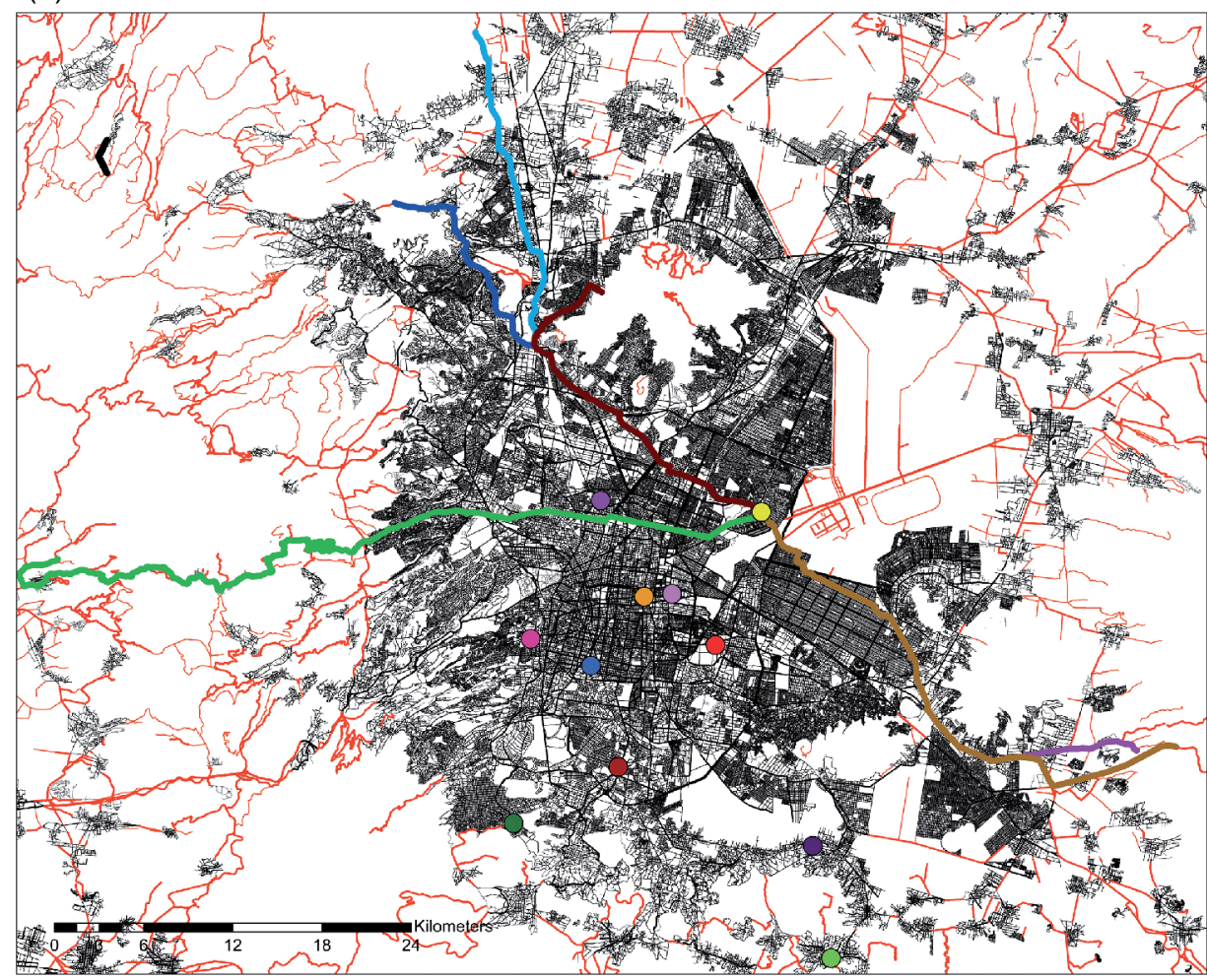

Rutas de disposición de RSU desde las ET de Gustavo A. Madero a los SDF en el Estado de México que reciben residuos de la Ciudad de México (b)

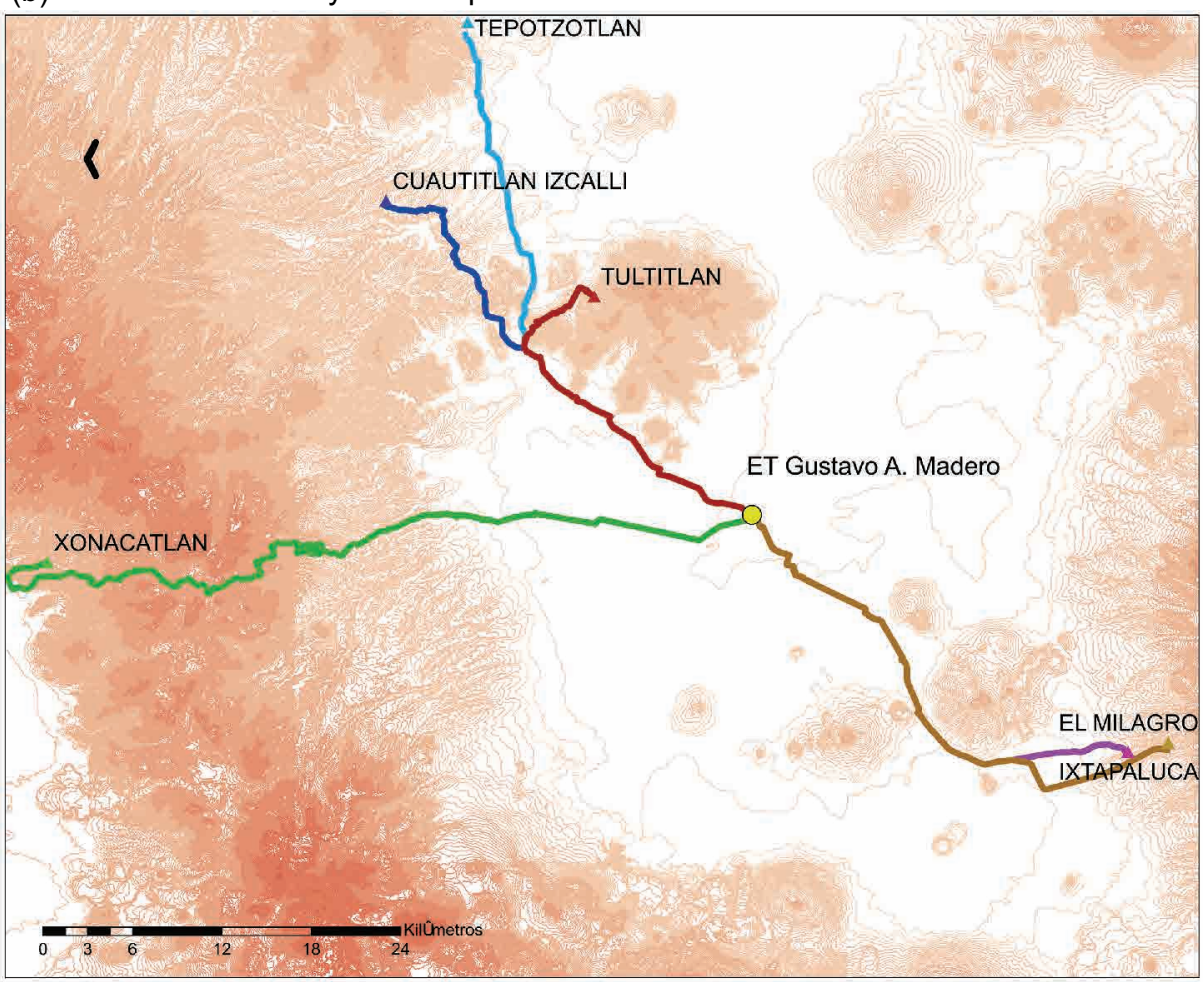

ET

Alvaro Obregón

- Azcapotzalco

Benito Juárez

- Central de Abastos

Coyoacán

Cuauhtémoc

$\bigcirc$ Gustavo A. Madero

- Iztapalpa

Milpa Alta

Tlalpan

Venustiano Carranza

- Xochimilco

CALLES

TIPO

Calle de cuarto orden

- Calle de primer orden

Calle de segundo orden

- Calle de tercer orden

- Carretera

- Terracería
Fig. 5. (a) Red de nodos y vertices (esquinas y calles) y rutas generadas a partir del análisis de redes en SIG, desde la estación de transferencia (ET) Gustavo A. Madero a los sitios de diposición final (SDF) en el Estado de México. (b) Rutas generadas usando la herramienta de análisis de redes en SIG, desde la ET Gustavo A. Madero a los SDF en el Estado de México 
CUADRO IV. DISTANCIAS CALCULADAS, USANDO LA HERRAMIENTA ANÁLISIS DE REDES, DESDE CADA ET DE LA CIUDAD DE MÉXICO HACIA CADA SDF EN EL ESTADO DE MÉXICO.

\begin{tabular}{|c|c|c|c|c|c|}
\hline ET & SDF & $\begin{array}{c}\text { Distancia calculada } \\
\text { usando la herramienta } \\
\text { Análisis de redes } \\
(\mathrm{km})\end{array}$ & ET & SDF & $\begin{array}{l}\text { Distancia calculada } \\
\text { usando la herramienta } \\
\text { Análisis de redes } \\
(\mathrm{km})\end{array}$ \\
\hline \multirow{6}{*}{$\begin{array}{l}\text { Álvaro } \\
\text { Obregón** }\end{array}$} & Xonacatlán & 54.02 & \multirow{6}{*}{$\begin{array}{l}\text { Gustavo A. } \\
\text { Madero }\end{array}$} & Xonacatlán & 64.32 \\
\hline & Cuautitlán Izcalli & 37.06 & & Cuautitlán Izcalli & 37.34 \\
\hline & Tepotzotlán & 45.46 & & Tepotzotlán & 44.35 \\
\hline & Tultitlán & 27.62 & & Tultitlán & 27.82 \\
\hline & Ixtapaluca y la Cañada & 42.22 & & Ixtapaluca y la Cañada & 34.55 \\
\hline & El Milagro & 45.42 & & EI Milagro & 38.32 \\
\hline \multirow{6}{*}{ Azcapotzalco } & Xonacatlán & 53.85 & \multirow{6}{*}{ Iztapalapa } & Xonacatlán & 65.55 \\
\hline & Cuautitlán Izcalli & 29.07 & & Cuautitlán Izcalli & 44.00 \\
\hline & Tepotzotlán & 36.08 & & Tepotzotlán & 51.02 \\
\hline & Tultitlán & 19.16 & & Tultitlán & 34.48 \\
\hline & Ixtapaluca y la Cañada & 42.25 & & Ixtapaluca y la Cañada* & 30.44 \\
\hline & El Milagro & 45.92 & & El Milagro & 33.62 \\
\hline \multirow{6}{*}{$\begin{array}{l}\text { Benito } \\
\text { Juárez }\end{array}$} & Xonacatlán & 58.72 & \multirow{6}{*}{ Milpa Alta } & Xonacatlán & 110.77 \\
\hline & Cuautitlán Izcalli & 39.56 & & Cuautitlán Izcalli & 94.37 \\
\hline & Tepotzotlán & 47.17 & & Tepotzotlán & 101.50 \\
\hline & Tultitlán & 30.64 & & Tultitlán & 84.96 \\
\hline & Ixtapaluca y la Cañada & 38.30 & & Ixtapaluca y la Cañada & 40.27 \\
\hline & El Milagro & 41.53 & & El Milagro* & 39.89 \\
\hline \multirow{6}{*}{$\begin{array}{l}\text { Central de } \\
\text { abastos }\end{array}$} & Xonacatlán & 65.55 & \multirow{6}{*}{ Tlalpan } & Xonacatlán & 117.08 \\
\hline & Cuautitlán Izcalli & 44.00 & & Cuautitlán Izcalli & 50.51 \\
\hline & Tepotzotlán & 51.02 & & Tepotzotlán & 58.27 \\
\hline & Tultitlán & 34.48 & & Tultitlán & 41.74 \\
\hline & Ixtapaluca y la Cañada* & 30.44 & & Ixtapaluca y la Cañada & 45.80 \\
\hline & El Milagro & 33.62 & & El Milagro & 47.22 \\
\hline \multirow{6}{*}{ Coyoacán } & Xonacatlán & 63.57 & \multirow{6}{*}{$\begin{array}{l}\text { Venustiano } \\
\text { Carranza }\end{array}$} & Xonacatlán & 59.52 \\
\hline & Cuautitlán Izcalli & 46.61 & & Cuautitlán Izcalli & 37.90 \\
\hline & Tepotzotlán & 54.71 & & Tepotzotlán & 44.91 \\
\hline & Tultitlán & 37.70 & & Tultitlán & 28.37 \\
\hline & Ixtapaluca y la Cañada & 38.58 & & Ixtapaluca y la Cañada & 34.13 \\
\hline & El Milagro & 40.06 & & EI Milagro & 37.74 \\
\hline \multirow{6}{*}{ Cuauhtémoc } & Xonacatlán & 57.95 & \multirow{6}{*}{ Xochimilco } & Xonacatlán & 79.05 \\
\hline & Cuautitlán Izcalli & 37.29 & & Cuautitlán Izcalli & 61.41 \\
\hline & Tepotzotlán & 44.17 & & Tepotzotlán & 68.94 \\
\hline & Tultitlán & 27.63 & & Tultitlán & 52.40 \\
\hline & Ixtapaluca y la Cañada & 36.01 & & Ixtapaluca y La Cañada* & 26.96 \\
\hline & EI Milagro & 39.65 & & El Milagro & 28.48 \\
\hline
\end{tabular}

Nota: durante las encuestas no se obtuvo información del SDF al que se enviaban los RSU de la ET Álvaro Obregón.

ET: estaciones de transferencia, SDF: sitios de diposición final

*De acuerdo con las encuestas, sí envían sus residuos al SDF más cercano

Se muestran en negritas: el SDF al que se enviaban los RSU desde cada ET, de acuerdo con las encuestas y según el inventario 2012 de la SEDEMA; la distancia más corta calculada

de México, contribuyen a la saturación pronta de los SDF ubicados en este último, por lo que están disminuyendo su tiempo de vida útil, ya que ninguno fue diseñado para recibir grandes cantidades de residuos. Por esta razón sería apropiado encontrar sitios para futuros SDF colindantes con la ciudad, cuya capacidad sea adecuada para dar cabida a los $\mathrm{RSU}$ de varios municipios. 
CUADRO V. SDF DEL ESTADO DE MÉXICO MÁS CERCANOS A CADA ET, SEGÚN EL CRITERIO DE DISTANCIA MÍNIMA, OBTENIDOS MEDIANTE EL ANÁLISIS DE REDES

\begin{tabular}{llll}
\hline ET & SDF y distancia $(\mathrm{km})$ & ET & SDF y distancia $(\mathrm{km})$ \\
\hline 1. Álvaro Obregón & Tultitlán $(27.62)$ & 7. Gustavo A. Madero & Tultitlán (27.82) \\
2. Azcapotzalco & Tultitlán $(19.16)$ & 8. Iztapalapa & Ixtapaluca y La Cañada (30.44) \\
3. Benito Juárez & Tultitlán (30.64) & 9. Milpa Alta & Ixtapaluca y La Cañada (39.89) \\
4. Central de abastos & Ixtapaluca y La Cañada (30.44) & 10. Tlalpan & Tultitlán (41.75) \\
5. Coyoacán & Tultitlán (37.70) & 11. Venustiano C. & Tultitlán (28.37) \\
6. Cuauhtémoc & Tultitlán (27.63) & 12. Xochimilco & Ixtapaluca y La Cañada (29.97) \\
\hline
\end{tabular}

ET: estaciones de transferencia, SDF: sitios de diposición final

El SIG resultó de gran utilidad para calcular rutas y distancias. Los resultados sugieren que se están recorriendo distancias muy largas para llevar los residuos sólidos desde las ET de la ciudad a los SDF foráneos; esto, aunado al hecho de que depositarlos en lugares que no fueron diseñados para recibir grandes cantidades de RSU es una medida insostenible. Debido a que la etapa de transporte puede representar hasta el $70-80 \%$ de los costos totales de un sistema de manejo de residuos, una de las prioridades del gobierno local debería ser el reordenamiento de las rutas ET-SDF con el fin de reducir costos de traslado y hacer más eficiente el transporte, independientemente de otras medidas de manejo para la reducción de residuos en la ciudad que ayuden a resolver el problema de raíz.

\section{CONCLUSIONES}

Mediante el uso de SIG se logró la visualización de los flujos de RSU desde las estaciones de transferencia de la Ciudad de México hacia distintos sitios de disposición final en el Estado de México. El análisis de redes en SIG es una herramienta útil para el cálculo de rutas y estimación de distancias planimétricas entre puntos de una red de caminos, pero es necesario llevar a cabo un proceso de depuración y enriquecimiento de datos que permita generar una red con la información necesaria (tipos de caminos, sentido de calles, jerarquía, etc.) con el fin de que el software pueda calcular la ruta de un nodo a otro.

Respecto al análisis espacial se obtuvo que de las doce ET en operación en la Ciudad de México, sólo cuatro (Central de Abasto, Iztapalapa, Milpa Alta y Xochimilco) realizaron la disposición de sus RSU en el SDF más cercano durante el periodo 2013-2014. Para estas ET, los SDF más cercanos son Ixtapaluca y La Cañada.
Por otro lado, se observó que en siete ET no se recorre una distancia óptima al SDF debido a que, habiendo SDF cercanos a dichas ET, los residuos son trasladados a SDF más lejanos. En general, se concluye que el SDF más cercano y adecuado desde el punto de vista de distancia óptima para la disposición de RSU provenientes de la Ciudad de México es el que se encuentra en Tultitlán, Estado de México. Sin embargo, este sitio actualmente se encuentra clausurado como SDF y sólo sirve como ET para residuos de dicho municipio que luego se llevan al SDF de Tepotzotlán. No obstante, Tultitlán podría considerarse un lugar adecuado para un nuevo SDF interestatal, dada su cercanía con ocho de las ET ya existentes. Esto requeriría un convenio entre el gobierno de la Ciudad de México y el Estado de México, así como estudios técnicos para la selección de un sitio adecuado, independientemente de otras medidas de manejo para la reducción de residuos en la ciudad que ayuden a resolver el problema desde el punto de vista de la jerarquía del manejo de residuos.

La optimización de las rutas de transporte de RSU, que es indispensable para evitar la generación de gastos innecesarios, puede realizarse mediante SIG, ya que permite la visualización de cifras y datos junto con su localización. Esto, aunado al análisis espacial, puede generar nueva información sobre rutas y distancias. El presente estudio puede completarse con datos de costos de traslado, lo que daría como resultado una herramienta importante para la toma de decisiones.

\section{AGRADECIMIENTOS}

Este trabajo fue apoyado por la Secretaría de Investigación y Posgrado (SIP) del Instituto Politécnico Nacional. La investigación se llevó a cabo en la 
Escuela Nacional de Ciencias Biológicas (proyecto SIP201603) y la Unidad Profesional Interdisciplinaria de Biotecnología (proyecto SIP20144650). Los autores agradecen también a la Comisión de Operación y Fomento de Actividades Académicas (COFAA) del Instituto Politécnico Nacional, el apoyo recibido para la realización de este proyecto.

\section{REFERENCIAS}

Betanzo Q.E., Torres G.M.A., Romero N.J.A. y Obregón B.S.A. (2016). Evaluación de rutas de recolección de residuos sólidos urbanos con apoyo de dispositivos de rastreo satelital: análisis e implicaciones. Rev. Int. Contam. Ambie 32 (3), 323-337.

DOI: 10.20937/RICA.2016.32.03.07

Cardozo O.D., Gómez E.L. y Parras M.A. (2009). Teoría de grafos y sistemas de información geográfica aplicados al transporte público de pasajeros en Resistencia (Argentina). Transporte y Territorio 1, 89-111.

Ciudadanos en Red (2012). Rechazan en Izcalli paso de camiones con basura. Ciudadanos Contra la Corrupción A.C. [en línea]. http://ciudadanosenred.com.mx/rechazan-en-izcalli-paso-de-camiones-con-basura/ 15/01/2017

El Universal (2011). Confirma Ebrard cierre de Bordo Poniente. El Universal [en línea]. http://archivo.eluniversal.com.mx/notas/817180.html 13/01/2017

El Universal (2015). Las mentiras del GDF sobre el Bordo Poniente. El Universal [en línea]. https://www. eluniversal.com.mx/entrada-de-opinion/articulo/ jose-luis-luege-tamargo/metropoli/df/2015/11/23/lasmentiras-del-gdf 23/11/2015

Excélsior (2012). Riesgo de colapso en DF por basura por cierre de Bordo Poniente. Excélsior [en línea]. http://www.excelsior.com.mx/2012/01/09/comunidad/800345 13/01/2017

Hita R.A. (2009). Sistema de información geográfica (SIG) para la gestión de residuos sólidos urbanos (RSU) del municipio del Prat de Llobregat. Tesis de Licenciatura. Escuela Politécnica Superior de Construcción de Barcelona, Universidad Politécnica de Cataluña, Barcelona, España, 180 pp.

INECC-SEMARNAT (2012). Diagnóstico básico para la gestión integral de los residuos 2012. Informe. Instituto Nacional de Ecología y Cambio Climático-Secretaría de Medio Ambiente y Recursos Naturales, Ciudad de México, México, 201 pp.

INEGI (1998). Catálogo de símbolos y sus especificaciones para las cartas topográficas. Instituto Nacional de Estadística y Geografía [en línea]. http://www.inegi. org.mx/geo/contenidos/topografia/ 17/01/2017
Kanchanabhan J., Mohaideen J.A., Srinivasan S. y Sundaram V.L.K. (2010). Optimum municipal solid waste collection using geographical information system (GIS) and vehicle tracking for Pallavapuram municipality. Waste Manage. Res. 29 (3), 323-339.

DOI: $10.1177 / 0734242 \times 10366272$

La Jornada (2011a). Cierra en definitiva el Bordo Poniente. La Jornada [en línea]. http://www.jornada.unam. $\mathrm{mx} / 2011 / 12 / 20 /$ capital/031n1 cap 13/01/2017

La Jornada (2011b). La crisis de la basura, por el cierre anticipado de Bordo Poniente. La Jornada [en línea]. http://www.jornada.unam.mx/2011/12/28/ capital/029n1cap 14/01/2017

La Razón (2011). Cierre del Bordo Poniente provoca crisis de basura. La Razón de México [en línea]. http://www. razon.com.mx/spip.php?article104311 14/01/2017

Lámbarri V.F., Rivas T.L.A., Trujillo F.M. y Martínez S.J.A. (2015). La gestión de residuos sólidos en México. En: Residuos en Hispanoamérica: de lo ambiental a lo social (Martínez J.A., Bielsa R.O., Llopart García S. y Yesares N., Ed.). Universidad EAN, Bogotá, Colombia, pp: 117-151.

MVT (2013). Vecinos de Ixtapaluca protestan por paso de camiones recolectores de basura. MVT Agencia de noticias [en línea]. http://mvt.com.mx/vecinos-deixtapaluca-protestan-por-paso-de-camiones-recolectores-de-basura/14/01/2017

SEDEMA (2013). Inventario de residuos sólidos del Distrito Federal 2012. Informe. Secretaría de Medio Ambiente del Gobierno de la Ciudad de México, México, 136 pp.

SEDEMA (2015). Inventario de residuos sólidos de la Ciudad de México 2014. Informe. Secretaría de Medio Ambiente del Gobierno de la Ciudad de México, México, 139 pp.

SEDEMA(2017). Programa de gestión integral de residuos sólidos 2016-2020. Informe. Secretaría de Medio Ambiente del Gobierno de la Ciudad de México, México, 49 pp.

SEMARNAT (2003). Ley General para la Prevención y Gestión Integral de los Residuos. Secretaría de Medio Ambiente y Recursos Naturales, Ciudad de México, México, $53 \mathrm{pp}$.

SEMARNAT (2012). Curso virtual de gestión de residuos sólidos urbanos y de manejo especial. Secretaría de Medio Ambiente y Recursos Naturales [en línea]. http://aplicaciones.semarnat.gob.mx/residuos/login. php?going_to=index.php 27/11/2016

Sendra J., Jiménez A. y Díaz M. (2012). Sistemas de información geográfica y localización óptima de instalaciones y equipamientos. 2a ed. Alfaomega. Ciudad de México, México, 417pp. 